\title{
COMPRENSIÓN LECTORA Y REDACCIÓN EN ESTUDIANTES DE LA ESPECIALIDAD DE ESPAÑOL Y LITERATURA DE LA FACULTAD DE PEDAGOGÍA Y HUMANIDADES DE LA UNCP
}

\author{
Cerrón Lozano, Alberto y Pineda Lozano, Marilú
}

Facultad de Pedagogía y Humanidades de la Universidad Nacional del Centro del Perú

\begin{abstract}
RESUMEN
La investigación es aplicada y de nivel descriptivo. El método aplicado fue el descriptivo con un diseño descriptivo correlacional. Se investigó la comprensión lectora y su relación con el nivel de redacción de los estudiantes del I y IX semestres de la especialidad de Español y Literatura de la Facultad de Pedagogía y Humanidades de la Universidad Nacional del Centro del Perú. Se tuvo una muestra de 72 estudiantes a la que se aplicó una prueba de comprensión lectora así como instrumentos para medir el nivel de redacción de los mismos estudiantes. Los resultados nos mostraron la existencia de una correlación directa y positiva muy alta entre la comprensión lectora y la redacción en Ios alumnos del I semestre de la especialidad de Español y Literatura de la Facultad de Pedagogía y Humanidades de la Universidad Nacional del Centro del Perú, pues la $r$ de Pearson fue: $r=0,791$. Asimismo se determinó que existe una correlación directa y positiva alta entre la comprensión lectora y la redacción en los alumnos del IX semestre de la especialidad de Español y Literatura de la Facultad de Pedagogía y Humanidades de la Universidad Nacional del Centro del Perú, como se evidencia en el resultado de la $r$ de Pearson: $r=0,683$. Y como tercera conclusión se señaló que existe una relación directa y positiva alta entre el rendimiento en la comprensión lectora y la redacción en los alumnos del I y IX semestre de la especialidad de Español y Literatura de la Facultad de Pedagogía y Humanidades de la Universidad Nacional del Centro del Perú; tal como lo muestra el resultado: "r" de Pearson = 0,7197546.
\end{abstract}

Palabras clave: comprensión lectora, redacción

\section{READING COMPREHENSION AND WRITING IN STUDENTS OF THE SPECIALTY OF SPANISH AND LITERATURE OF THE FACULTY OF PEDAGOGY AND HUMAN UNCP}

\begin{abstract}
The research is applied and descriptive level. The method used was a correlational descriptive design. Reading comprehension was investigated and its relationship to the level of student writing of the I and IX semesters majoring in Spanish and Literature at the Faculty of Education and Humanities of the Universidad Nacional del Centro del Peru. There was a sample of 72 students to whom it was administered a reading comprehension test as well as tools for measuring the level of writing. The results showed us that there is a direct and high positive correlation between reading comprehension and writing in the I semester students majoring in Spanish and Literature at the Faculty of Education and Humanities of the Universidad Nacional del Centro del Peru, because the Pearson's $r$ was: $r=0.791$. It was also determined that there is a direct and high positive correlation between reading comprehension and writing in the ninth semester students majoring in Spanish and Literature at the Faculty of Education and Humanities of the Universidad Nacional del Centro del Peru, as evidenced the result of Pearson's $r: r=0.683$. And a third conclusion stated that there is a direct positive relationship between high performance in reading comprehension and writing in the students of I and IX semester majoring in Spanish and Literature at the Faculty of Education and Humanities of the Universidad Nacional del Centro del Peru, as shown by the result: $r$ Pearson $=0.7197546$.
\end{abstract}

Key words: reading, writing

\footnotetext{
* Este trabajo de investigación fue recibido el 20/12/2008 retornado para su revisión 26/10/2009 y aprobado para su publicación 16/11/2009

'Email: edmaore@yahoo.es

2Email: manuelortize@hotmail.com
} 


\section{INTRODUCCIÓN}

El desarrollo de la competencia lingüística que implica el logro de las capacidades de comprensión y producción de textos, es básica para que la persona pueda realizar exitosamente un trabajo intelectual; por ello conocer con precisión los niveles de comprensión lectora y redacción de los alumnos de la especialidad de Español y Literatura de la Facultad de Pedagogía y Humanidades de la UNCP y establecer la relación existente entre estos dos aspectos de la competencia comunicativa es de suma importancia, ya que dicha información permitirá orientar mejor el trabajo pedagógico hacia el desarrollo de la competencia lingüística de los alumnos que se forman para ser docentes.

En este contexto el problema que guió la investigación fue: ¿Qué relación existe entre la comprensión lectora y la redacción en los alumnos del I y IX semestre de la especialidad de Español y Literatura de la Facultad de Pedagogía y Humanidades de la Universidad Nacional del Centro del Perú? En función a ello el objetivo general del estudio fue establecer la relación que existe entre la comprensión lectora y la redacción en los estudiantes del I y IX semestre de la especialidad de Español y Literatura de la Facultad de Pedagogía y Humanidades de la Universidad Nacional del Centro del Perú. Los objetivos específicos fueron: a) Identificar el rendimiento en comprensión lectora en los alumnos del I y IX semestre de la especialidad de Español y Literatura de la Facultad de Pedagogía y Humanidades de la Universidad Nacional del Centro del Perú, b) Determinar el rendimiento en redacción en los estudiantes del I y IX semestre de la especialidad de Español y Literatura de la Facultad de Pedagogía y Humanidades de la Universidad Nacional del Centro del Perú.

La hipótesis planteada sostenía que existe una relación directa entre la comprensión lectora y la redacción en los estudiantes del I y IX semestre de la especialidad de Español y Literatura de la Facultad de Pedagogía y Humanidades de la Universidad Nacional del Centro del Perú.

El trabajo en cuestión se justifica en el hecho de que, se ha notado ciertas deficiencias en la comprensión de textos escritos y también en la capacidad de redactar textos -así lo muestran las observaciones empíricas- donde los estudiantes de la muestra de estudio (I y IX semestres de la especialidad de Español y Literatura), lo cual es preocupante, pues se trata de estudiantes que se forman para ser docentes.
Finalmente, el trabajo de investigación permitió identificar que existe relación directa entre las capacidades de comprensión lectora y redacción en los estudiantes de la muestra de estudio.

\section{MATERIAL Y MÉTODOS}

El estudio se realizó en una muestra de estudio a 72 estudiantes del I y IX semestres de la especialidad de Español y Literatura de la Facultad de Pedagogía y Humanidades de la UNCP del año académico 2006-I. La investigación fue de tipo básica y de nivel descriptivo. El método aplicado fue el descriptivo con un diseño descriptivo correlacional.

\section{RESULTADOS}

\section{Comprensión lectora y redacción en estudiantes del I semestre de Español y Literatura}

Cuadro 1. Resumen estadístico

\begin{tabular}{lcc}
\hline \multicolumn{1}{c}{ Estadígrafos } & Comprensión Lectora & Redacción \\
\hline MEDIA ARITMÉTICA & 12,26 & 10,45 \\
MEDIANA & 12,00 & 11,00 \\
MODA & 12,00 & 11,00 \\
PUNTAJE MÁXIMO & 16 & 14 \\
PUNTAJE MÍNIMO & 7 & 5 \\
RANGO & 9 & 9 \\
DESVIACIÓN ESTÁNDAR & 2,13 & 2,06 \\
COEFICIENTE DE VAR. & 17,34 & 19,74 \\
KURTOSIS & 0,169275831 & 0,78744915 \\
ASIMETRÍA & 0,371194695 & - \\
R DE PEARSON & \multicolumn{2}{c}{0,70382723} \\
\hline
\end{tabular}

Como se puede apreciar en el cuadro, los calificativos que se obtuvieron en la evaluación de comprensión lectora y redacción son ligeramente diferentes. Mientras que en la comprensión lectora los alumnos tienen una media aritmética de 12,26, en redacción es de10,45 (1,82 puntos más en comprensión lectora). Respecto a la mediana, en la comprensión lectora tienen 1,00 puntos más que en redacción diferencia que también se aprecia en la moda. 
El puntaje máximo en la comprensión lectora fue 16,00 mientras que en la redacción fue 14,00 (1,00 punto de diferencia) y respecto al puntaje mínimo en la comprensión lectora 07 mientras que en redacción 05 (2,00 puntos de diferencia). El rango que es el recorrido del menor al mayor puntaje, en la comprensión lectora y en redacción se tiene 9 respectivamente.

Respecto a la desviación estándar en la comprensión lectora fue 2,13 mientras que en redacción 2,06, el cual nos dice que los resultados obtenidos son un poco más homogéneos en la variable redacción; el cual también lo evidencia el coeficiente de variación, que en comprensión lectora es 17,34 y en redacción es 19,74.

Respecto a la Kurtosis, en la comprensión lectora fue 0,169 ; mientras que en redacción fue 0,787 , con el cual se deduce que la gráfica en ambos casos es estudiantes. Respecto a la asimetría de Pearson: en la comprensión lectora es 0,371 que significa asimetría a la derecha o positiva; y en redacción se tiene - 0,803 que significa asimetría negativa 0 a la izquierda.

En los resultados de la correlación entre comprensión lectora y redacción se tiene $r=0,791$, lo cual significa que existe una correlación directa y positiva muy alta entre la comprensión lectora y la redacción en los estudiantes del I semestre de la especialidad de Español y Literatura de la Facultad de Pedagogía y Humanidades de la Universidad Nacional del Centro del Perú.

\section{Comprensión lectora y redacción en estudiantes del IX ciclo de Español y Literatura}

Cuadro 2. Resumen estadístico

\begin{tabular}{lcc}
\hline \multicolumn{1}{c}{ Estadígrafos } & Comprensión Lectora & Redacción \\
\hline MEDIA ARITMÉTICA & 12,76 & 11,74 \\
MEDIANA & 13,00 & 12,00 \\
MODA & 14,00 & 12,00 \\
PUNTAJE MÁXIMO & 16 & 15 \\
PUNTAJE MÍNIMO & 8 & 8 \\
RANGO & 8 & 7 \\
DESVIACIÓN ESTÁNDAR & 2,51 & 1,64 \\
COEFICIENTE DE VAR. & 19,67 & 13,96 \\
KURTOSIS & $-0,948982394$ & 0,0260792 \\
ASIMETRÍA & $-0,281083169$ & $-0,4845981$ \\
R DE PEARSON & \multicolumn{2}{c}{0,683941014} \\
\hline
\end{tabular}

Como se puede apreciar en el cuadro resumen del IX semestre, los calificativos que se obtuvieron en la evaluación de comprensión lectora y redacción son algo diferentes. Mientras que en la comprensión lectora los alumnos del IX semestre tienen una media aritmética de 12,76, en redacción tienen 11,74 (1,03 puntos más en comprensión lectora). Respecto a la mediana, en la comprensión lectora tienen 13,00 , y en redacción tienen 12,00, (o sea 1,00 puntos más). Respecto a la moda en la comprensión lectora tienen 14,00 , y en redacción 12,00, o sea existen 2 puntos de diferencia.

El puntaje máximo en la comprensión lectora fue 16,00 mientras que en la redacción fue 15,00 (1,00 punto de diferencia) y respecto al puntaje mínimo en la comprensión lectora y redacción tienen 08 respectivamente. El rango en la comprensión lectora es 8 y en redacción 7 .

Respecto a la Desviación Estándar en la comprensión lectora fue 2,51 mientras que en redacción 1,64, el cual nos dice que los resultados obtenidos son un poco más homogéneos en la variable redacción; el cual también lo evidencia el Coeficiente de variación, que en comprensión lectora es 19,67 y en redacción es 13,96.

Respecto a la Kurtosis, en la comprensión lectora fue -0,948 que significa que la curva es platicúrtica ; mientras que en redacción fue 0,026 , del cual se deduce que la gráfica es leptocúrtica. Respecto a la asimetría de Pearson: en la comprensión lectora es -0,281 y en redacción -0,484 que significa en ambos casos una asimetría negativa 0 a la izquierda.

En la correlación entre comprensión lectora y redacción establecida a través de la $r$ Pearson se tiene: $r=$ 0,683 , lo que significa que existe una correlación directa y positiva alta entre la comprensión lectora y la redacción en los alumnos del IX semestre de la especialidad de Español y Literatura de la Facultad de Pedagogía y Humanidades de la Universidad Nacional del Centro del Perú.

\section{Prueba de hipótesis}

\section{Planteamiento de Hipótesis:}

- Hipótesis nula: $\mathrm{H}_{0}$ : No existe una relación directa entre la comprensión lectora y la redacción en los 
alumnos del I y IX semestre de la especialidad de Español y Literatura de la Facultad de Pedagogía y Humanidades de la Universidad Nacional del Centro del Perú.

- Hipótesis alterna: $H_{1}$ : Existe una relación directa entre la comprensión lectora y la redacción en los alumnos del I y IX semestre de la especialidad de Español y Literatura de la Facultad de Pedagogía y Humanidades de la Universidad Nacional del Centro del Perú.
- Nivel de significancia o riesgo: Es de: $a=0,05$.

El estadígrafo de prueba: El estadígrafo de Prueba más apropiado para este caso es la " $r$ " de Pearson.

Cálculo del estadístico de prueba:

$$
r=\frac{n \sum x y-\sum x \sum y}{\sqrt{n \sum x^{2}-\left(\sum x\right)^{2}\left[n \sum y^{2}-\left(\sum y\right)^{2}\right.}}
$$

Cuadro 3. Coeficiente de Correlación

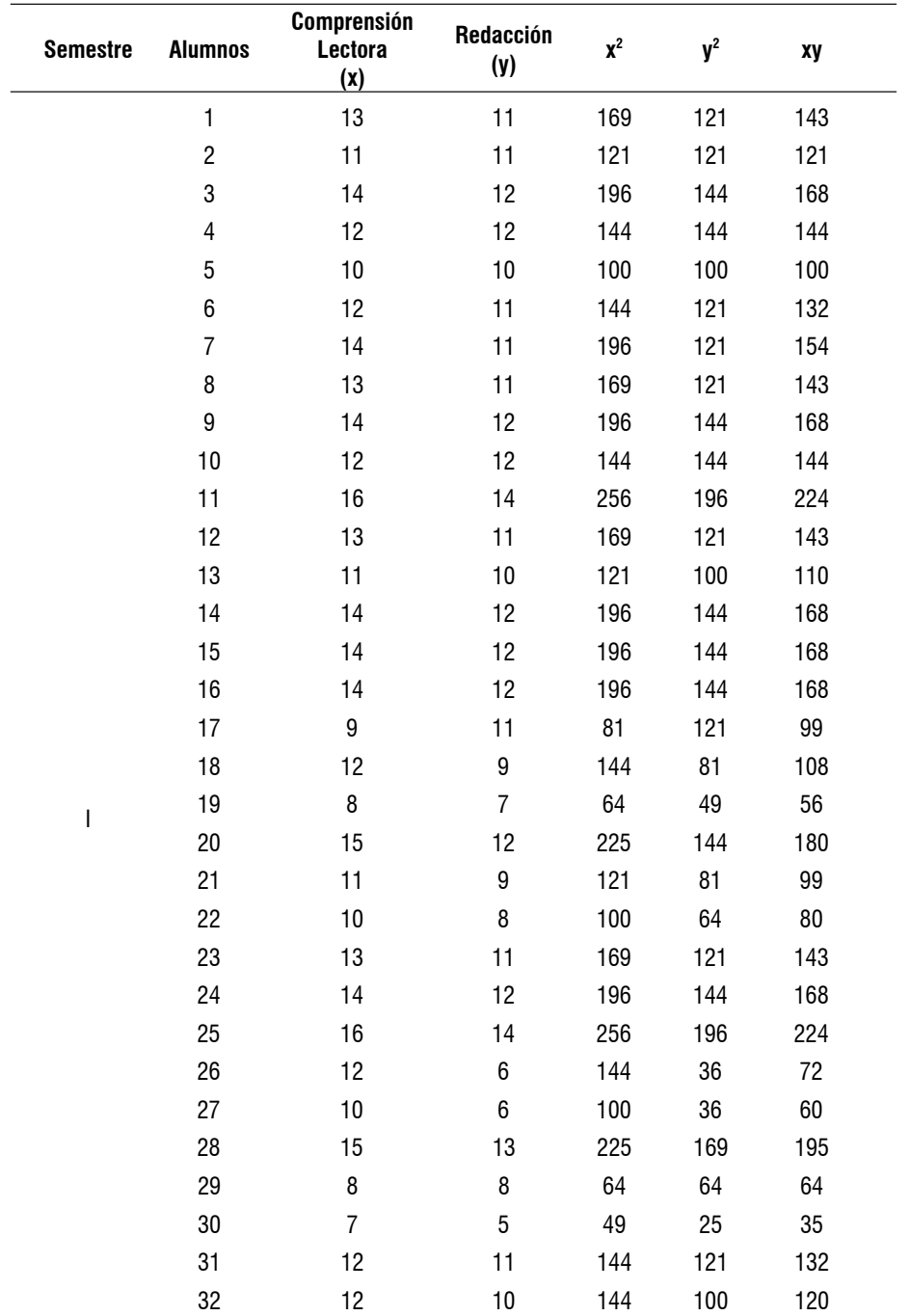




\begin{tabular}{|c|c|c|c|c|c|c|}
\hline & 33 & 11 & 10 & 121 & 100 & 110 \\
\hline & 34 & 13 & 9 & 169 & 81 & 117 \\
\hline & 35 & 14 & 11 & 196 & 121 & 154 \\
\hline & 36 & 12 & 10 & 144 & 100 & 120 \\
\hline & 37 & 13 & 11 & 169 & 121 & 143 \\
\hline & 38 & 12 & 10 & 144 & 100 & 120 \\
\hline & 39 & 16 & 12 & 256 & 144 & 192 \\
\hline & 40 & 14 & 12 & 196 & 144 & 168 \\
\hline & 41 & 9 & 12 & 81 & 144 & 108 \\
\hline & 42 & 16 & 12 & 256 & 144 & 192 \\
\hline \multirow[t]{30}{*}{ IX } & 43 & 10 & 11 & 100 & 121 & 110 \\
\hline & 44 & 15 & 12 & 225 & 144 & 180 \\
\hline & 45 & 9 & 10 & 81 & 100 & 90 \\
\hline & 46 & 12 & 12 & 144 & 144 & 144 \\
\hline & 47 & 16 & 14 & 256 & 196 & 224 \\
\hline & 48 & 8 & 10 & 64 & 100 & 80 \\
\hline & 49 & 13 & 12 & 169 & 144 & 156 \\
\hline & 50 & 16 & 15 & 256 & 225 & 240 \\
\hline & 51 & 14 & 13 & 196 & 169 & 182 \\
\hline & 52 & 16 & 14 & 256 & 196 & 224 \\
\hline & 53 & 15 & 12 & 225 & 144 & 180 \\
\hline & 54 & 11 & 12 & 121 & 144 & 132 \\
\hline & 55 & 10 & 12 & 100 & 144 & 120 \\
\hline & 56 & 14 & 13 & 196 & 169 & 182 \\
\hline & 57 & 14 & 12 & 196 & 144 & 168 \\
\hline & 58 & 16 & 13 & 256 & 169 & 208 \\
\hline & 59 & 11 & 10 & 121 & 100 & 110 \\
\hline & 60 & 12 & 11 & 144 & 121 & 132 \\
\hline & 61 & 13 & 10 & 169 & 100 & 130 \\
\hline & 62 & 14 & 13 & 196 & 169 & 182 \\
\hline & 63 & 10 & 10 & 100 & 100 & 100 \\
\hline & 64 & 12 & 11 & 144 & 121 & 132 \\
\hline & 65 & 13 & 10 & 169 & 100 & 130 \\
\hline & 66 & 15 & 12 & 225 & 144 & 180 \\
\hline & 67 & 12 & 10 & 144 & 100 & 120 \\
\hline & 68 & 14 & 15 & 196 & 225 & 210 \\
\hline & 69 & 13 & 14 & 169 & 196 & 182 \\
\hline & 70 & 9 & 8 & 81 & 64 & 72 \\
\hline & 71 & 8 & 9 & 64 & 81 & 72 \\
\hline & 72 & 14 & 11 & 196 & 121 & 154 \\
\hline TOTAL & & 900 & 796 & 11630 & 9076 & 10183 \\
\hline
\end{tabular}

\section{Toma de decisiones:}

Como la "r" de Pearson = 0,7197546; con lo cual se concluye que existe una relación directa y positiva alta entre el rendimiento en la comprensión lectora y la redacción en los alumnos del I y IX semestre de la especialidad de Español y Literatura de la Facultad de Pedagogía y Humanidades de la Universidad Nacional del Centro del Perú; con lo cual se prueba la hipótesis en toda su extensión y significado con un $95 \%$ de significancia. 


\section{DISCUSIÓN}

En los últimos 25 años la investigación en comprensión y producción del discurso escrito ha evolucionado a través de caminos separados y de perspectivas teóricas divergentes. Sin embargo, generalmente se tiende a pensar que un buen comprendedor tiende a ser un buen escritor de textos y consecuentemente un lector deficiente tiende a desempeñarse pobremente como escritor. Esta idea señala la existencia de algún tipo de conocimiento común entre escritura y lectura. Los resultados de la presente investigación en alguna medida corroboran esta idea, pues el resultado de la $r$ de Pearson= 0,7197546 señala que existe correlación directa y positiva alta entre el rendimiento en la comprensión lectora y la redacción en los alumnos del I y IX semestre de la especialidad de Español y Literatura de la Facultad de Pedagogía y Humanidades de la Universidad Nacional del Centro del Perú.

\section{CONCLUSIÓN}

- Existe una correlación directa y positiva muy alta entre la comprensión lectora y la redacción en los alumnos del I semestre de la especialidad de Español y Literatura de la Facultad de Pedagogía y Humanidades de la Universidad Nacional del Centro del Perú, pues la $r$ de Pearson fue: $r=0,791$.

- Existe una correlación directa y positiva alta entre la comprensión lectora y la redacción en los alumnos del IX semestre de la especialidad de Español y Literatura de la Facultad de Pedagogía y Humanidades de la Universidad Nacional del Centro del Perú, como se evidencia en el resultado de la $r$ de Pearson: $r=0,683$,

- Existe una relación directa y positiva alta entre el rendimiento en la comprensión lectora y la redacción en los alumnos del I y IX semestre de la especialidad de Español y Literatura de la Facultad de Pedagogía y Humanidades de la Universidad Nacional del Centro del Perú; tal como lo muestra el resultado: " $r$ " de Pearson $=0,7197546$.

\section{LITERATURA CITADA}

ALLENDE, F. y CONDEMARíN, M. 1986. La lectura: Teoría, evaluación y desarrollo. Segunda edición, Santiago de Chile: Edit. Andrés Bello.

ARISTIZABAL, A. 1991. Cómo leer mejor. Tercera edición, Colombia: Universidad de Antioquia.

BAUMANN, J. 1990. La comprensión lectora (cómo trabajar la idea principal en el aula). Madrid: Visor Distribuciones S.A.

CAIRNEY, T. 1999. Enseñanza de la comprensión lectora. Tercera edición, Madrid: Ediciones Morata.

COOPER, D. 1990. Cómo mejorar la comprensión lectora. Madrid: Visor Distribuciones S.A.

DE VEGA, M. y otros 1990. Cómo mejorar la comprensión lectora. Madrid: Visor Distribuciones S.A.

PINZÁS, J. 1995. Leer pensando. Lima- Perú: Asociación de Investigación Aplicada y Extensión Pedagógico.

SOLÉ, I. 2000. Estrategias de lectura, 11ava.edición, Barcelona: Edit. Grao. 